\title{
Non-equilibrium interface of a two-dimensional low-temperature crystal
}

\author{
V.A. Shneidman*, K.A. Jackson, K.M. Beatty \\ Arizona Materials Laboratory, Department of Materials Science and Engineering, The University of Arizona, Tucson, AZ 85721, USA
}

Received 13 August 1999; accepted 23 December 1999

Communicated by S.R. Coriell

\begin{abstract}
We study the interface in an Ising system with nearest-neighbor interaction on a square lattice at very low temperatures, when the Wulff shape of a nucleus is almost a perfect square. Spins are randomly flipped via Metropolistype dynamics. At moderately strong undercoolings, the step nucleation rates can be evaluated from the first principles. This permits the description of the growth of an infinite interface using a step-on-step nucleation picture. The averaged shape of the interface is universal (i.e., it does not depend on any parameters as long as the interface remains stable), and its growth rate, in appropriate variables, also has no free parameters. For finite sizes of two-dimensional crystals their growth can be dominated by nucleation of single steps, and becomes size-dependent. For both infinite- and finite-size interfaces growth rates are in good agreement with large-scale Monte Carlo simulations. At high undercoolings the interface becomes very rough, in which case the crystals switch to circular shapes, in contrast to the equilibrium Wulff expectation. (C) 2000 Elsevier Science B.V. All rights reserved.
\end{abstract}

PACS: 81.10.Aj; 68.35.Ct; 02.70.Lq; 05.50. + q

Keywords: Step nucleation; Roughening; Ising model; Simulations

\section{Introduction}

By now it is well known $[1,2]$ that the dynamics of crystal growth and crystal morphology are crucially determined by the structure of crystal-liquid or crystal-vapor interface. In the classical paper [3], Burton et al. suggested roughening of the crystal-vapor interface at some temperature, $T_{\mathrm{R}}$, although they estimated the latter as being above the

\footnotetext{
* Corresponding author. Present address: Department of Physics, NJIT, Newark, NJ 07102, USA. Tel.: + 1-973-5963555; fax: + 1-973-596-5794.

E-mail address: vitaly@oak.njit.edu (V.A. Shneidman).
}

melting point, $T_{\mathrm{m}}$. Subsequently, Jackson [4] extended these ideas to melt growth and showed that for a large number of materials (or, face orientations) $T_{\mathrm{R}}$, in fact, falls below $T_{\mathrm{m}}$. Scaling-type relations which depend on a simple combination of the entropy of fusion $\Delta S$ (Boltzmann constant is taken as unity) and a geometric factor which depends on the face orientation can be established [4], allowing one to predict the type of growth for a given material or orientation. For vapor growth of molecular crystals $[5,6]$ with variable $\Delta S$ it is possible to take the same crystal from below to above its roughening temperature, with dramatic changes in the observed crystal morphologies. 
Problems of growth below and above the roughening transition, as well as the transition itself also stimulated more mathematically oriented studies [7-10] (see also citations in Section 3). Monte Carlo (MC) simulations, originally initiated for the solid-on-solid model [11-13] provided an important complement to both mathematical and experimental work.

In the present work, we will be concerned with a seemingly simpler situation of growth of a cold two-dimensional "crystal", which can be considered as model of an island on a flat face below $T_{\mathrm{R}}$ (nucleation of such islands provides the only possibility for three-dimensional growth, in the absence of defects). The one-dimensional interface is always above the corresponding "roughening temperature" which is strictly zero [7]. Nevertheless, as will be discussed below, at small $T$ growth will proceed precisely as if the interface were smooth, namely via nucleation and growth of steps. Only for very large values of $\Delta S$, when the nucleation barrier to step nucleation virtually disappears, does the interface become rough.

In certain features our study will proceed as a two-dimensional analog of Gilmer's work [13] on three-dimensional growth via nucleation of islands on flat faces. There are certain technical differences in the MC model and in the analytical description (due to a different dimension) which will be described below. A more important difference, however, comes from the fact that the two key issues of the higher dimensional description, namely the island nucleation rate and their growth and interaction of Kolmogorov-Avrami type, inevitably are approximations. While the Kolmogorov-Avrami picture for the two-dimensional Ising model seems to be rather accurate $[14,15]$, the accuracy of the standard nucleation rate predictions is often unsatisfactory [16]. In fact, the only way to comply with MC results for a two-dimensional interface is to scale the time with the nucleation rate, in effect treating the latter as an adjustable parameter [13].

In contrast, one-dimensional nucleation of steps can be described exactly at low temperatures. The corresponding analog of the Kolmogorov-Avrami description also seems to be rigorously justified for the nearest-neighbor interactions between atoms.
This provides a unique possibility of clarifying the growth picture through a direct comparison of analytics with MC simulation data. It seems worth mentioning that an independent study of the interface growth in a two-dimensional Ising model has been initiated recently by Rikvold and co-workers (see the Appendix of Ref. [15]). That study deals with the orientational dependence of the growth rate of an interface at higher temperatures for somewhat different spin flip dynamics. Presumably, in contrast to our study, the interface in that work is initially rough, although the details still have to be published [17].

Our paper is organized as follows. In Section 2 the parameters of the MC model (which will play the role of "experiment") are described and the main notations are introduced. Typical results of MC simulations are presented in Section 3 which also discusses possible relations to earlier studies. Section 4 contains analytical evaluation of the step nucleation rate, and a semi-analytical description of the interface growth via the step-on-step nucleation mechanism. Effects of finite size of a growing crystal are also considered in this section, and results are compared with MC simulations. A brief discussion concludes the study.

\section{Main parameters of the model and notations}

In applications of the Ising model to crystallization problems due to Jackson, Gilmer and Tëmkin (JGT) the up and down orientations of a spin are associated with the solid and liquid phases, respectively (the more complicated two-component case [18] will not be discussed here). The down-up spin flip rates are controlled by a single parameter, $\Delta S$, related to the entropy of fusion discussed above

$\beta_{0}=\exp (-\Delta S)$

The up-down flips are more complicated, and depend on the resulting change in the interaction energy with the surrounding spins. In the current realization of the JGT model only interaction between neighboring up ("solid") spins is nonzero, and is determined by the bond energy, $\phi$. Any other nearest-neighbor interaction on the same square lattice can be mapped onto this case. Flipping of 
a spin with breaking of a single bond will proceed at a rate

$\alpha=\exp (-\phi / T)$.

Similarly, breaking of two bonds results in a rate of $\alpha^{2}$, etc. For the low temperatures considered, however, such rates are vanishingly small compared to $\alpha$.

Equilibrium is achieved for $\Delta S_{\text {eq }}=2 \phi / T$. Otherwise, the difference $2 \phi / T-\Delta S$ determines the "reduced undercooling", $\delta S$, and will represent the main controlling parameter of the problem. In that way analogies can be established with Ising model simulations in magnetic systems (see, e.g. Ref. [15] and references therein) where $T \delta S / 2$ sould be replaced by the magnetic field. Technically, a variable $\Delta S$ corresponds to crystal growth from vapor where this quantity changes with pressure at a given temperature; a variable $T$ with a fixed entropy of fusion is more appropriate for melt growth. Our primary goal, however, is to be able to predict the interface growth for an arbitrary pair of $T$ and $\delta S$, and a choice of a particular parameter as the primary variable is just a matter of convention.

We will be mainly concerned with the domain of parameters

$\delta S \gg 1, \quad 2 \phi / T-\delta S>1$

which is equivalent to

$\alpha^{2} \ll \alpha, \beta_{0} \ll 1$

and which implies a region of deep undercoolings, though still far from absolute instability, with a small probability of flipping of a single down spin. The main dimensionless parameter will be defined as

$\kappa \equiv \alpha / \beta_{0}=\exp (\phi / T-\delta S)$.

This parameter determines the ratio of the up-down and down-up flipping rates of a single spin near a flat interface, and determines the step nucleation rate (see Section 4.1). For large, though nonasymptotic values of $\kappa \gg 1$ the interface remains flat, growing via nucleation of steps - see Section 4.2. Alternatively, for smaller $\kappa$ there is practically no barrier to step nucleation, with dramatic changes in the structure of the interface. In the following, time will be scaled with $\beta_{0}$, the "arrival rate":

$t^{\prime}=t \beta_{0}$.

In this notation the growth rate of an individual step of length $l$ in the direction parallel to a flat interface, becomes especially simple

$\mathrm{d} l / \mathrm{d} t^{\prime}=2$.

Thus, the growth rate of a flat interface is expected to have the same value as the number of kinks (approximately twice the number of steps), and this will lead to simplification of other expressions as well.

\section{The Monte Carlo simulations}

The temperature for most of the simulations was chosen as $\phi / 15$, which is about 0.12 of the twodimensional critical temperature. At such cold temperatures the equilibrium shape of a crystal is close to a perfect square [19]. Using the zero-temperature values of the interfacial energy, $\sigma=\phi / 2$, one estimates the side of a critical square to be

$m_{*}=\phi / T \delta S$.

The initial "crystal" was prepared as a circular island of up spins with a diameter of 50 lattice vectors. This is noticeably larger than $m_{*}$ since values of $\delta S$ of at least several units were considered, and there were no chances for the crystal to reverse its growth and decay.

Characteristic shapes of the growing crystals are shown in Fig. 1. At smaller undercoolings the crystal almost immediately (on the scale of the characteristic growth time) acquires an equilibrium square shape which persists during further growth. For higher undercoolings, however, the interface turns unstable with respect to the addition of single spins. Here the interface acquires a characteristic very rough structure, while the shape of the crystal is circular, in contrast to equilibrium expectations. It is worth noting that change in the shape of an island formed by a growing screw dislocation was observed in early MC studies by Gilmer [12] using the solid-on-solid model. This change was associated, however, with the changing temperature 

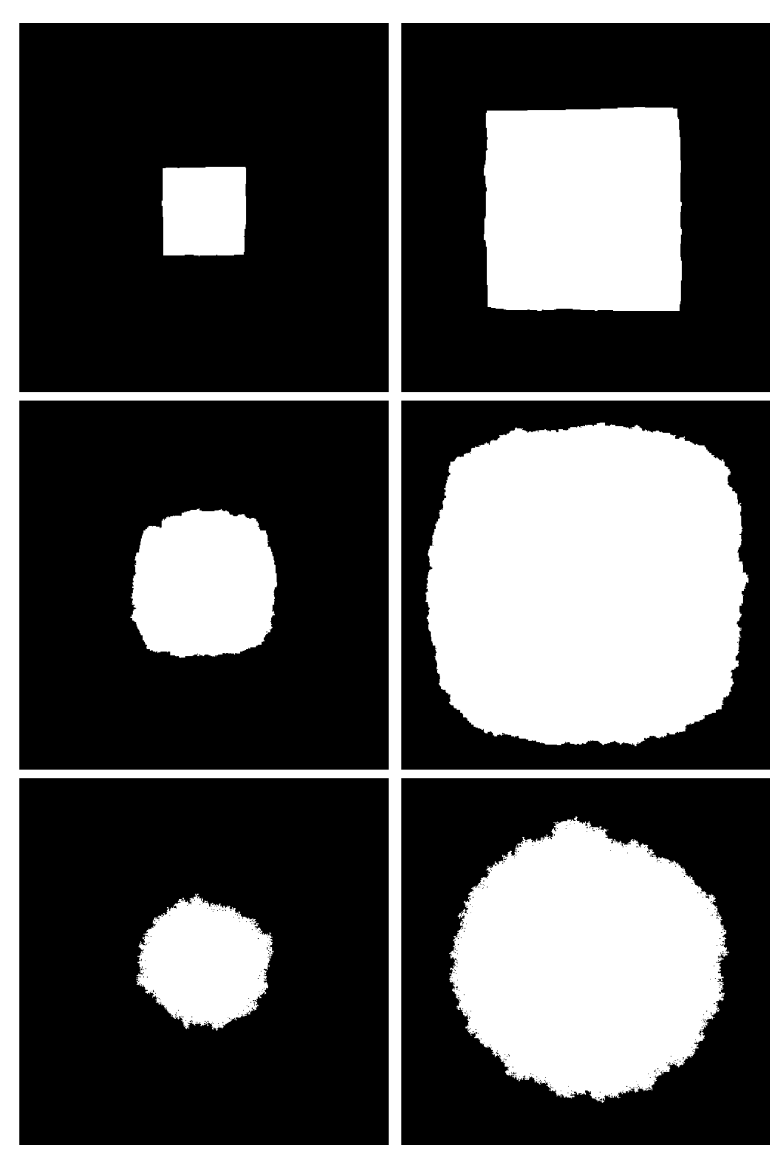

Fig. 1. Typical snapshots of crystal shapes from smaller (left) to larger (right) times for three different undercoolings: $\delta S=8$ (upper row), $\delta S=12$ (middle) and $\delta S=20$ (down). In each case the initial shape was a circle with diameter of 50 spins $\left(\frac{1}{10}\right.$ of the size of the simulation system, which is the black square). At the smaller undercooling a few kinks on the otherwise flat faces of the square are seen (for still smaller $\delta S$ the square would look practically perfect). At large $\delta S$ the interface becomes unstable and very rough (lower row), and the equilibrium square shape is not achieved.

(which would be observed even at equilibrium in accord with the Wulff construction [19]). In our case the temperature is held fixed; modification of shape in this case is an essentially nonequilibrium effect, due to an abrupt loss of the interface stability.

Transitions between a circular shape and the one reflecting the lattice symmetry had been observed in models of aggregation processes [20-24]; roughening transition also can be of exceptional interest here. As a rule, dynamics in those models is not dictated by detailed balance, making it extremely difficult (in our terms) to give a quantitative definition of "temperature" or "undercooling". Nevertheless, similarity of shape transformations is remarkable, probably reflecting some general universality features of strongly nonequilibrium dynamics.

Change in morphology of growing two-dimensional crystals is also observed experimentally for monolayers of surfactants at the surface of an aqueous solution [25]. The minor variation of temperature results in a strong change in the supersaturation, which is analogous to our changing undercooling. While the physical situation is somewhat different (growth from solutions is more adequately described by a conserved-type MC dynamics [18]), the similarity is nevertheless noteworthy.

During simulations the growth rate of an interface, $U$, was obtained from the number of spins belonging to the crystal, with the correction for its actual shape. Alternatively, an initially flat interface was considered, and results were tested for consistency. Two types of the spin flip dynamics [14] were employed. The full spin flip dynamics allows flipping of any spin throughout the entire simulation domain. It is rather slow for low temperatures, but it is also closest to the real physical picture, allowing not only nucleation of steps but also formation of new crystals in the bulk of the "liquid". Alternatively, a "truncated" dynamics which forbids an individual up flip (and forbids the reverse process to comply with detailed balance) was considered. In practice, this restricts the spin flipping events to the vicinity of the interface, tremendously accelerating a simulation run. Compared to the solid-on-solid model, the truncated dynamics still allows for overhangs and vacancies, which can become appreciable for a rough interface.

Results for the two types of the spin flip dynamics completely agree at small undercoolings. At moderately large undercoolings results agree at small times until occasional coagulation with a newly nucleated crystal (possible only for the full dynamics) changes the growth pattern. For very large undercoolings, when nucleation rates of new 
crystals are too fast, only the truncated description of an interface is possible.

A typical simulated system was $500 \times 500$ square (the outside black square in Fig. 1), and simulation runs were performed on a local Silicon Graphics workstation. The boundary conditions are unimportant as long as the inside crystal in Fig. 1 does not exceed the external dimension. Comparative studies of a $1000 \times 200$ box of spins with a flat interface in the long dimension and periodic boundary conditions were performed. Occasionally, larger systems of $4 \times 10^{6}$ spins were considered to cope with size-dependent growth effects (see Section 4.4). Such runs were performed at the University of Arizona Supercomputer.

\section{Theory}

\subsection{The step nucleation rate on a flat interface}

For $\kappa \gtrsim 1$ nucleation of a step is a rare event. This means that nucleation on top of a newly formed step can be neglected, as long as this step remains short. Thus, one can write a system of Becker-Döring-type [26,27] equations for the step distributions, $f_{l}$ normalized per unit length of the interface, with $l$ being the length of a step,

$\mathrm{d} f_{l} / \mathrm{d} t=I_{l}-I_{l+1}$.

Here $t$ is the new time (we omit the prime) and $I_{l}$ are the fluxes,

$I_{1}=1-\kappa f_{1}$,

$I_{2}=2 f_{1}-2 \kappa \alpha f_{2} \approx 2 f_{1}$,

$I_{3}=2 f_{2}-2 \kappa \alpha f_{3} \approx 2 f_{2}$,

etc. The value of $\kappa \alpha$ is small away from phase equilibrium, which allows the above simplification in Eqs. (11) and (12): the possibility of loosing a spin is negligible for every step, except the one with the smallest size.

There is one essential difference, however, in Eqs. (9)-(12) compared to those of Becker and Döring. The latter are usually written for higher dimensions and represent an approximate model of the nucleation process. In contrast, the one-dimensional nucleation is expected to be described exactly at low temperatures by the above equations. Otherwise, the solution of Eqs. (9)-(12) proceeds along the standard lines [26].

The steady-state solution corresponds to a constant, size-independent flux, $I_{1}=I_{2}=\cdots \equiv I$, with $I$ being the step nucleation rate. One obtains

$I=\frac{1}{1+\kappa / 2} \approx 2 / \kappa$

Transient nucleation effects which can be important for two-dimensional Ising nucleation [14] are of limited interest for the present case due to extremely small values of the size of a "critical step". This is shown in more detail in Appendix A.

\subsection{Growth of an infinite interface}

While the step-on-step nucleation can be neglected when the underlying steps have just nucleated, such secondary nucleation becomes important after those steps grow, occupying a noticeable fraction of their layer. This fraction, $L_{n}$ ( $n$ being the number of a layer), can be estimated using a one-dimensional version of the Kolmogorov-Avrami description as

$L_{n}=1-\exp \left(-L_{n}^{\mathrm{ext}}\right)$.

Here $L_{n}^{\text {ext }}$ is the "extended" fraction, which would be occupied by the steps in the absence of their overlap. Again, while the Kolmogorov-Avrami approach is a model for interaction between nuclei at higher dimensions, in the one-dimensional lowtemperature case it becomes virtually exact. Indeed, interacting steps grow unperturbed, except for the points of immediate contact.

It should be emphasized that such a description allows one to evaluate the crystallized fraction in the $n$th layer averaged over its entire length. Upon this averaging the information about the actual distribution of crystallized segments in each layer (thus, information about roughness) is lost. In particular, the number of kinks has to be evaluated separately, as will be discussed in the next section. 
Since steps on the $n$th level can appear only due to lower-level nucleation, one has for the extended fraction

$L_{n}^{\mathrm{ext}}=I \int_{0}^{t} L_{n-1}(\tau) l(t-\tau) \mathrm{d} \tau$

with $l(t) \simeq 2 t$ being the size of a growing step.

Introducing temporarily another new "time"

$\tau=t \sqrt{I \mathrm{~d} l / \mathrm{d} t}$

one has

$L_{n}^{\mathrm{ext}}(\tau)=\int_{0}^{\tau} L_{n-1}\left(\tau^{\prime}\right)\left(\tau-\tau^{\prime}\right) \mathrm{d} \tau^{\prime}$

or

$\frac{\mathrm{d}^{2} L_{n}^{\mathrm{ext}}}{\mathrm{d} \tau^{2}}=L_{n-1}$

(In a similar three-dimensional problem of filling in successive layers by nucleation and growth of islands one encounters a chain of differential equations of the third order [13]; those lead to oscillatory transient behavior which is absent in the present case - see below.)

With $L_{0} \equiv 1$ and initial conditions at $\tau=0$

$L_{n}=0, \quad \mathrm{~d} L_{n} / \mathrm{d} \tau=0, \quad n \geqslant 1$.

Eqs. (14) and (18) (which are rather straightforward for numerical realization) determine the full system of equations which describe the layered growth. They can be cast exclusively in terms of the "physical" fractions, $L_{n}$,

$\frac{\mathrm{d}^{2} L_{n}}{\mathrm{~d} \tau^{2}}+\frac{1}{1-L_{n}}\left(\frac{\mathrm{d} L_{n}}{\mathrm{~d} \tau}\right)^{2}=\left(1-L_{n}\right) L_{n-1}$.

At $\tau \rightarrow \infty$ this system is expected to have a kinklike solution

$L_{n}(\tau)=L(n-u \tau) T_{n}$

with $T_{n}$ being some periodic function of $n$ with a period 1 (which is not of much interest in the present context). The constant $u$ in Eq. (21) is the interface growth rate - the key parameter of the problem. It is crucial that Eq. (20) is nonlinear: one can show that the linearized version for $L_{n} \rightarrow 0$ is satisfied by $\exp [-a(n-u \tau)]$ with $u=\mathrm{e}^{a / 2} / a$ for any $a$. (In other words, a linear system does not

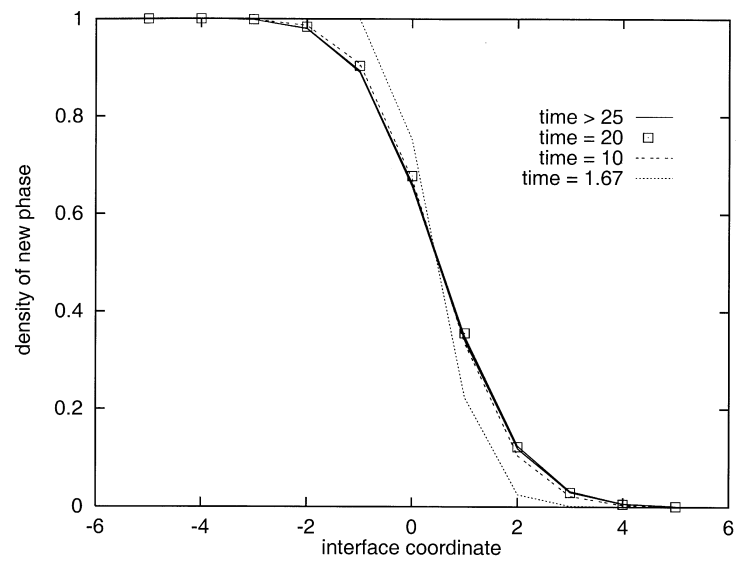

Fig. 2. Transient shapes of the interface as a function of $n-\bar{n}$ for near-integer values of $\bar{n}$ from the step-on-step nucleation picture for different values of the scaled "time", $\tau$. The asymptotic shape at $\tau \gtrsim 20$ is universal, and is not expected to depend either on temperature or on undercooling, as long as the interface remains flat. Cusps are due to discrete nature of $n$ and are not a plotting artifact.

approach a universal asymptotic regime, keeping the memory of specific initial conditions.)

At present we have been unable to find analytically a solution of type (21). This can be easily done numerically, however. Once $L_{n}(\tau)$ are obtained, location of the interface is determined as

$\bar{n}(\tau)=\sum_{n=1}^{\infty} L_{n}(\tau)$

The mean-field shape of the interface calculated as a function of $n-\bar{n}$ for near-integer values of $\bar{n}$ approaches an asymptotic limit after about 20 layers are grown - see Fig. 2. The mean-field width of the interface, $\Delta$, is determined as

$\Delta^{2}(\tau)=\sum_{n=1}^{\infty} 2 n L_{n}(\tau)-[\bar{n}(\tau)]^{2}-\bar{n}(\tau)$

and approaches the value of $1.31 \ldots$ as $\tau \rightarrow \infty$. (The physical width is, of course, larger due to local fluctuations within one layer which are averaged out when evaluating $L_{n}$.)

At large times the growth rate, $\mathrm{d} \bar{n} / \mathrm{d} \tau$, approaches a constant value of

$u=1.354 \ldots$. 


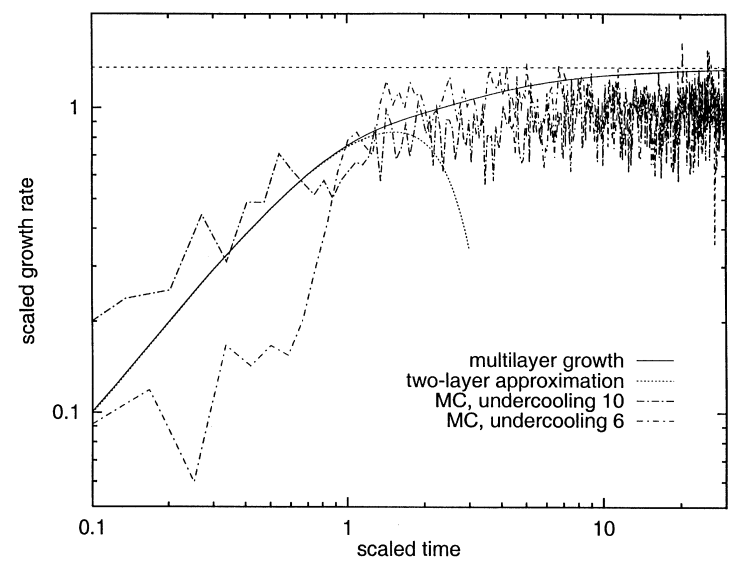

Fig. 3. Scaled growth rate of an interface, $\mathrm{d} \bar{n} / \mathrm{d} \tau$ from Eqs. (14) and (18) (solid line) and from MC simulations as a function of the scaled time, $\tau$, Eq. (16). No matching parameters were used. The asymptotic value (dashed line) is $u \approx 1.354$. Dotted line is the analytical solution for the first two layers (Appendix B). Note that MC runs for two very different undercoolings produce close results if cast in the scaled variables.

This is seen from Fig. 3 where the logarithmic scale is used to highlight the initial, nonasymptotic regime (Eq. (20) can be integrated analytically for $n=1,2-$ see the Appendix B - giving the dotted line in Fig. 3).

Note that the values $u$ and $\Delta$, as well as the mean-field shape of the interface are "universal" - they do not depend either on the physical parameters of the problem (temperature or undercooling) or on the initial conditions from which growth is started. (The latter was also tested numerically by switching to nonzero initial conditions for the first layer, $L_{1}$.) Similarly, the transient behavior does not depend on any parameters if expressed as a function of the scaled time, $\tau$. This scaling is expected to hold in the general case as well, even if some information about the structure of the interface was lost in the mean-field description. Indeed, as seen from Fig. 3, two very different MC runs (with nonscaled time different by more than 50 times) practically collapse onto each other if Eq. (16) is employed.

Returning to the "primary" time, Eq. (6), one obtains the interface growth rate

$U=1.915 \sqrt{I} \approx 2.709 / \sqrt{\kappa}$.

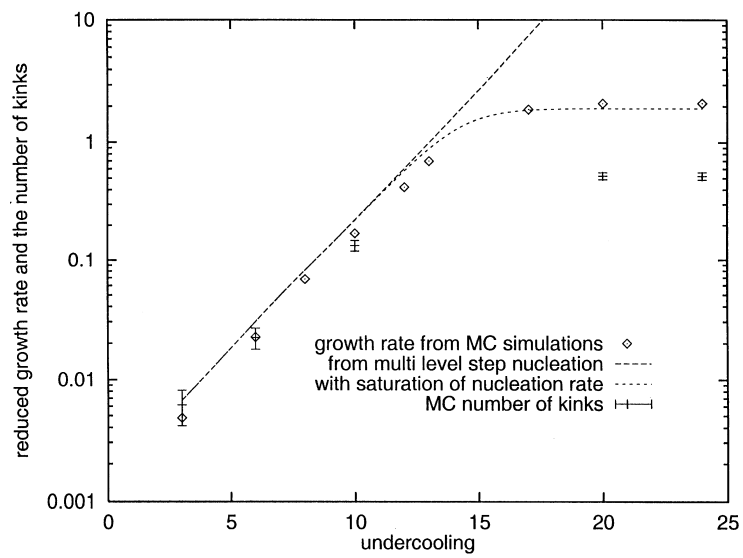

Fig. 4. Reduced growth rate $U$ (growth rate reduced by the arrival rate, $\beta_{0}$ ) and the number of kinks as a function of $\delta S$ from MC simulations (diamonds and errorbars) and the growth rate from the model predictions (lines). Straight long dashed line - Eq. (25) with asymptotic expression for the step nucleation rate, $I \approx 2 / \kappa$. Short dashed line - the same equation with $I=2 /(\kappa+2)$ which saturates at large $\delta S(\kappa \rightarrow 0)$.

This dependence is in good agreement with MC simulations in Fig. 4.

Formally, the case of large undercoolings where the step nucleation rate reaches its extreme and saturates (and where the interface is anything but flat - see Fig. 1) is beyond the step-on-step nucleation description. Nevertheless, since the main effect, the saturation of the number of kinks for a rough interface is described by the above expression, it remains reasonable in the region of large $\delta S$ as well. This is seen from Fig. 4, although the somewhat surprising numerical accuracy in the rough region could be partly coincidental.

\subsection{Kink density}

The kink density per unit length, $\rho_{k}$, can be calculated in the MC study by evaluating the number of atoms near the interface with exactly two solid bonds. The densities are shown in Fig. 4 for several selected undercoolings. Scatter (error bars) is due to the finite length of the interface and decreases with the number of kinks.

Recall that in the selected time units, with the attachment rate at the kink site being equal to unity, the growth rate $U$ is expected to coincide 
with the kink density. This indeed seems to be the case at moderate undercoolings, see Fig. 4. For larger $\delta S$ (rough interface) growth can start at any point and is larger than the number of kinks.

For a rough interface the kink density approaches the value of $0.5(0.52 \pm 0.03)$ and is insensitive to the value of $\delta S$. It also does not seem to change much with temperature, being equal to $0.51 \pm 0.04$ when the latter was increased by $50 \%$.

As mentioned, the semi-analytical, mean-fieldtype description of the previous section does not allow an independent estimation of the number of kinks (just as in higher dimensions the Kolmogorov-Avrami approach gives the total crystallization volume but not the complicated shape of the interface formed by the overlapping nuclei). Once $U$ is evaluated, however, the kink density is expected to be close to $U$, just as in the MC case. Alternative estimations described below can be useful to get a feeling of the uncertainty.

Using a Kolmogorov-like estimation of the number of nucleated steps (which is twice the number of kinks), one obtains

$\rho_{k} \approx 2 I \int_{0}^{\infty} \mathrm{d} t \exp \left(-I t^{2}\right)=\sqrt{\pi I}$

which is virtually indistinguishable from the growth rate $U$ in Eq. (25).

Another possibility is to estimate $\rho_{k}$ from the fact that nucleation of steps effectively proceeds during a time interval $1 / U$ which is required to cover one layer. This gives $\rho_{k} \approx \sqrt{2 I} / u, u=1.35 \ldots$, which is about two times smaller and determines the lower boundary of the estimation.

\subsection{Growth of finite crystals}

The total nucleation rate on the perimeter of a square with side $m$ is given by

$I_{\mathrm{tot}}=4 m I$.

If the square is sufficiently small, nucleation of a single step will limit the growth. Since a step will stop when it reaches the edge, one needs four nucleation events to complete a new layer. The corre- sponding time is given by

$\Delta t_{\mathrm{s}} \sim 4 / I_{\mathrm{tot}}=1 / \mathrm{Im}$

which leads to a size-dependent growth rate

$\frac{\mathrm{d} m}{\mathrm{~d} t}=2 m I$

This gives the side of a square exponentially increasing with time

$m(t)=m_{0} \exp (2 I t) \approx m_{0} \exp (4 t / \kappa)$

with $m_{0}$ being the initial side of the square (note that $m_{0}$, though small, is expected to be much larger than the "critical square", discussed in Section 3). Crossover, the transition to multi-step nucleation regime is expected to take place at

$m \gtrsim 1 / \sqrt{8 I} \approx \sqrt{\kappa} / 4$.

At larger $m$ growth rate is expected to coincide with that of a flat interface.

Results of simulations for two small undercoolings (where finite-size effects are the strongest) are shown in Fig. 5. Agreement with Eq. (30) is reasonable (no matching parameters were used), although the latter slightly overestimates the size at larger times, probably, due to neglected crossover to multi-step nucleation.

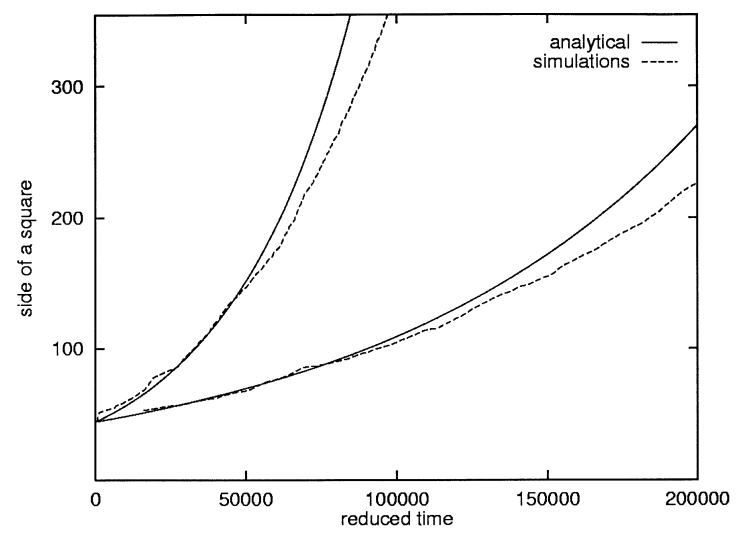

Fig. 5. Growth of squares via single-step nucleation at small undercoolings $\delta S=3$ (upper) and $\delta S=2$ (lower). Analytical results (solid lines) are from Eq. (30). Dashed lines are the MC simulations. Time is $t^{\prime}$ from Eq. (6). 


\section{Discussion}

The central part of the present study was the description of growth of a two-dimensional crystal via step nucleation on its interface. An interesting point is that such growth takes place well above the mathematical "roughening transition", which for a one-dimensional interface is strictly at $T=0$ [7]. Nevertheless, at sufficiently low temperatures the distance between steps on the interface is exponentially large, and growth will proceed in exact analogy with a flat surface. In other words, mathematical "roughness" can be viewed as an equilibrium property of an infinite interface, while a finite interface (or a finite stretch of one) can look perfectly "smooth" and should be treated accordingly when growth is considered.

Roughening of the interface at cold temperatures is still possible - see Fig. 1. However, the crossover becomes a strictly nonequilibrium phenomenon which takes place at such large undercoolings that the interface is close to the loss of stability (i.e. any surface site, and not only a kink, becomes admissible). The instability leads to an unusual structure of the interface, almost a dendrite-like, which normally would not be associated with a system with a nonconserved order parameter. Naturally, roughening is accompanied by the change in the morphology of a crystal, from an almost a perfect square to a circle; this also can be understood in terms of the orientation-dependent growth velocity [28].

The analytical results of the step-on-step nucleation picture could be identified with those obtained from large scale Monte Carlo (MC) simulations without any matching parameters - see Figs. 3 and 4. This possibility is unique for the two-dimensional crystal where the one-dimensional step nucleation can be described exactly. A minor deviation from the $\mathrm{MC}$ results can be noted, nevertheless, for the interface growth rate in Fig. 4. It could be that the step-on-step nucleation picture still can be further refined (e.g., by taking into account the possibility of disappearance of an already nucleated step). It is also possible that the quest for very cold temperatures - which leads to "crystal clear" pictures of the corresponding shapes, as in Fig. 1 - can lead to a certain abuse of the random number generator [16], leading to a minor loss of numerical accuracy.
While understanding of the above deviations can be of mathematical interest, from the point of any reasonable experimental accuracy there is an excellent agreement between the step-on-step nucleation picture and simulations. Of further interest is the possibility of scaling, when in appropriate variables the results (e.g., the interface location) turn out to be a "universal" function of a properly scaled time. Rather unexpectedly, the averaged shape of the interface, Fig. 2, does not depend on any parameters at all. While this prediction could not be confirmed or disproved in the MC studies, its credibility is indirectly supported by the accuracy of the step-on-step nucleation description in every other situation. To comply with the intuitive picture of an interface recall that the amount of solid phase in a layer is averaged over its entire length. As long as the interface remains smooth, the ratio of kinks on subsequent layers remains the same (even if their separation within each layer changes), leading to the same dependence of the density of the solid phase as a function of the interface parameter.

Finally, it was possible to consider finite-size effects in the growth of a two-dimensional crystal. Besides being more physically realistic, description of a corresponding closed interface has a technical advantage of being insensitive to the boundary conditions imposed on the Monte Carlo model. (For a straight interface a periodic boundary condition is normally used, but there always remain questions about the consequences of such a choice [9].) At small times finite-size squares grow with time exponentially, and the increment could be identified with the Monte Carlo predictions, again with no matching parameters, as in Fig. 5.

\section{Conclusion}

Growth of a two-dimensional crystal in a strongly undercooled "liquid" was considered using large scale Monte Carlo simulations. The shape of such a crystal may or may not correspond to the equilibrium Wulff shape, depending on the degree of the undercooling.

At moderate undercoolings growth proceeds via nucleation of one-dimensional steps, as for a 
smooth interface, despite the fact that the system is above the equilibrium roughening transition. Quantitative description of the step-on-step nucleation becomes virtually exact in the low-dimensional case considered, and is in good agreement with the Monte Carlo results.

\section{Appendix A. Transient effects in step nucleation}

One needs to consider only the distribution of the smallest steps with $l=1$, since larger steps grow deterministically. One has from Eqs. (9)-(11),

$\mathrm{d} f_{1} / \mathrm{d} t=1-(\kappa+2) f_{1}$

or

$f_{1}(t)=f_{1}^{\mathrm{st}}(1-\exp [-t(\kappa+2)])$

with $f_{1}^{\text {st }}=1 /(\kappa+2)$ being the steady-state value of $f_{1}$. The characteristic time to establish a steady state at the smallest sizes is thus given by a small value $1 /(\kappa+2)$. On the other hand, the times of physical interest (the time to complete a new layer - see Section 4.2) are of the order of $1 / \sqrt{I}=$ $\sqrt{\kappa+2}$. The relative importance of transient nucleation effects is thus given by

$(\kappa+2)^{-3 / 2}$.

This is a small number in the smooth region $(\kappa \gg 1)$; it increases as roughening is approached $(\kappa \lesssim 1)$, but remains smaller than unity.

\section{Appendix B. Two-layer approximation for the growth rate}

Eqs. (15) and (17) can be integrated analytically for $n=1,2$. The interface location at small times can be approximated by Eq. (22) with summation truncated at $n=2$. The corresponding growth rate in this approximation is given by

$$
\begin{aligned}
\mathrm{d} \bar{n} / \mathrm{d} \tau= & \mathrm{e}^{-\tau^{2} / 2}\left\{\tau+\exp \left[\mathrm{e}^{-\tau^{2} / 2}-1\right.\right. \\
& \left.\left.+\frac{\sqrt{\pi}}{2} \tau \operatorname{erf}\left(\frac{\tau}{\sqrt{2}}\right)\right]\left[\tau-\operatorname{erf}\left(\frac{\tau}{\sqrt{2}}\right)\right]\right\}
\end{aligned}
$$

and is shown by a dotted line in Fig. 3. The expression can be useful since it accurately describes a large part of the transient period, although the asymptotic growth regime is approached well after the first two layers are filled, and requires a full consideration.

\section{References}

[1] A.A. Chernov, Modern Crystallography III, Crystal Growth, Springer, Berlin, 1984.

[2] P. Hartman, in: I. Sunagawa (Ed.), Morphology of Crystals, Part A, Terra Sci, Tokyo, 1987, p. 269.

[3] W.K. Burton, N. Cabrera, F.G. Frank, Philos. Trans. Roy. Soc. 243 A (1951) 299.

[4] K.A. Jackson, Can. J. Phys. 36 (1958) 683.

[5] K.A. Jackson, C.E. Miller, J. Crystal Growth 40 (1977) 169.

[6] X.Y. Liu, P. Bennema, J. Crystal Growth 139 (1994) 179.

[7] J.D. Weeks, G.H. Gilmer, H.J. Leamy, Phys. Rev. Lett. 31 (1973) 549.

[8] J.D. Weeks, The roughening transition, in: I. Riste (Ed.), Ordering in Strongly Fluctuating Condensed Matter Systems, Plenum, New York, 1980, pp. 293-317.

[9] C. Rottman, M. Wortis, Phys. Rep. 103 (1984) 59.

[10] U. Alon, M.R. Evans, H. Hinrichsen, D. Mukamel, Phys. Rev. Lett. 76 (1996) 2746.

[11] H.J. Leamy, K.A. Jackson, J. Appl. Phys. 42 (1971) 2121.

[12] G.H. Gilmer, J. Crystal Growth 36 (1976) 15.

[13] G.H. Gilmer, J. Crystal Growth 49 (1980) 465

[14] V.A. Shneidman, K.A. Jackson, K.M. Beatty, Phys. Rev. B 59 (1999) 3579.

[15] R.A. Ramos, P.A. Rikvold, M.A. Novotny, Phys. Rev. B 59 (1999) 9053.

[16] V.A. Shneidman, K.A. Jackson, K.M. Beatty, J. Chem. Phys. 111 (1999) 6932.

[17] P.A. Rikvold, M. Kolesik, J. Stat. Phys., submitted for publication.

[18] K.A. Jackson, G.H. Gilmer, D.E. Temkin, Phys. Rev. Lett. 75 (1995) 2530.

[19] C. Rottman, M. Wortis, Phys. Rev. B 24 (1981) 6274.

[20] D. Richardson, Proc. Cambridge Philos. Soc. 74 (1973) 515.

[21] R. Savit, R. Ziff, Phys. Rev. Lett. 55 (1985) 2515.

[22] P. Meakin, Phys. Rev. A 38 (1988) 418.

[23] P. Meakin, Phys. Rep. 235 (1993) 189.

[24] T. Halpin-Healy, Y.C. Zhang, Phys. Rep. 254 (1995) 215.

[25] B. Berge, L. Faucheux, K. Schwab, A. Libchaber, Nature 350 (1991) 322.

[26] L. Farkas, Z. Phys. Chem. 25 (1927) 236.

[27] R. Becker, W. Döring, Ann. Phys. 24 (1935) 719.

[28] P. Devillard, H. Spohn, Europhys. Lett. 17 (1992) 113. 